\title{
Clinical Analysis of Bevacizumab plus FOLFIRI Regimen as Front-Line Therapy for Chinese Patients with Advanced Colorectal Cancer
}

\author{
Xijian Zhou ${ }^{1}$, Jun Yang ${ }^{2,3^{*}}$, Jinying Liang ${ }^{2}$, Peng $\mathrm{Li}^{2}$, Yuquan $\mathrm{Wu}^{4}$, Xiaoqiang Fan ${ }^{1}$, Xiangyong $\mathrm{Li}^{1}$ \\ ${ }^{1}$ Department of Hematology \& Oncology, 101 Hospital of PLA, Wuxi, China; ${ }^{2}$ College of Pharmacy, Xinxiang Medical University, \\ Xinxiang, China; ${ }^{3}$ Kamp Institute for Medical Research, Changsha, China; ${ }^{4}$ Deaprtment for Senior Cadres, 117 Hospital of PLA, \\ Hangzhou, China. \\ Email: *bcd2009@126.com
}

Received July 12 ${ }^{\text {th }}, 2011$; revised August $16^{\text {th }}, 2011$; accepted August 25 $5^{\text {th }}, 2011$.

\begin{abstract}
The study was designed to evaluate the therapeutic and side effects of Bevacizumab plus FOLFIRI regimen as front-line therapy for Chinese patients with advanced colorectal cancer. A total of 15 previously untreated patients with advanced colorectal cancer received Bevacizumab plus FOLFIRI regimen as font-line therapy, in detail, irinotecan $180 \mathrm{mg} / \mathrm{m}^{2}$ was given intravenously on day1, then calcium folinate (CF) $200 \mathrm{mg} / \mathrm{m}^{2}, F$-fluorouracil (5-Fu) $400 \mathrm{mg} / \mathrm{m}^{2}$ given in bolus immediately after CF, day 1 - 2; 5-Fu $600 \mathrm{mg} / \mathrm{m}^{2}$ given continuously after bolus for 22 hours on day 1, day 2; Bevacizumab was given intravenously at dosage of $5 \mathrm{mg} / \mathrm{kg}$, on day-1. Therapeutic and side effects were evaluated at least after four cycles of treatment. The results showed that all the cases among the group were valid for response evaluation, with $C R 0, P R 10, S D$ 3, and $P D 2$. The response rate is $66.7 \%$ and median time to progression ( $m T T P$ ) was 10.6 months. The main toxicities were bone marrow suppression, nausea and vomiting, stomach pain and diarrhea; there was no chemotherapy-related death. The data suggested that the combination regimen with Bevacizumab plus FOLFIRI regimen was effective as front-line therapy for Chinese patients with advanced colorectal cancer, and the side effects were tolerable and manageable.
\end{abstract}

Keywords: Bevacizumab, Chinese Patients, FOLFIRI Regimen, Front-Line Therapy, Colorectal Cancer

\section{Introduction}

Colorectal cancer is one of the most common types of malignant tumor in China; the rate of new cases has been increasing these years. When found, it turned out to be the advanced colorectal cancer and required systemic treatment. Irinotecan is a topoisomerase I inhibitor, in the United States plus with Fluorouracil as the first-line treatment of advanced colorectal cancer. In our country, the domestic Irinotecan came into market later than domestic Oxaliplatin, so it is now more acceptable to patients with Oxaliplatin-based first-line treatment, Irinotecan-based program for second-line, and gradually become front-line [1].

The combination therapy of Irinotecan and Fluorouracil was previously carried out by giving the Fluorouracil in bolus with the IFL program. With the usage of in-depth study of Fluorouracil, it turned out that the therapeutic index of Fluorouracil continuous infusion is superior to intravenous injection, current clinical multi-Fluorouracil continuous infusion of FOLFIRI regimen was recommended as a way of both combined with the administration program $[2,3]$. FOLFIRI is a chemotherapy regimen for treatment of colorectal cancer. It is made up of the following drugs: FOL-folinic acid (leucovorin), a vitamin B derivative used as a "rescue" drug for high doses of the drug methotrexate and that modulates/potentiates/ reduces the side effects of fluorouracil; F-fluorouracil (5-FU), a pyrimidine analog and antimetabolite which incorporates into the DNA molecule and stops synthesis; and IRI-irinotecan (Camptosar), a topoisomerase inhibitor, which prevents DNA from uncoiling and duplicating. The clinical use of new drugs is one of the most important progressions in advanced colorectal cancer therapy, which obviously ameliorate the prognosis of the patients with advanced colorectal cancer. Cetuximab, a monoclonal antibody to epidermal growth factor receptor, is sometimes added to FOLFIRI. 
Bevacizumab is an new vessel anti-tumor drug, which has not been understand well in the combination with other anti-tumor drugs treating Chinese patients with advanced colorectal cancer. The study was designed to study the therapeutic effects after the treatment with Bevacizumab plus FOLFIRI regimen with Irinotecan as the basic drug in 15 Chinese patients with advanced colorectal cancer from December 2005 to December 2007 in our hospitals.

\section{Materials and Methods}

\subsection{General Materials}

15 patients, 8 male patients, 7 female patients age 37 - 65, average age 46.7, PS score 0 - 1, 10 colon carcinoma, 5 rectal cancer. The final diagnosis by pathohistological method were as follows: 3 poorly differentiated adenocarcinoma, 5 moderately differentiated adenocarcinoma, 3 mucinous adenocarcinoma, 4 signet-ring cell carcinoma, 5 hepatic metastasis, 2 celiac lymph nodes metastasis, 3 pulmonary metastasis, 1osseous metastasis and 4 multi-organ metastasis were examined by chest $\mathrm{x}$-ray, $\mathrm{CT}$, and type-B ultrasonic. They all had measurable focus of infection and were initial treatment cases. Expect life cycle were more than 3 months. The clinical routine symptoms and signs as: blood, urine and faeces, hepatic and renal function, electrocardiogram, CXR and/or CT were tested before treatment, contraindication were excluded.

\subsection{Treatment Programs}

Irinotecan hydrochloride (Jiangsu Hengrui Pharmaceutical Company, Trade Name: Aili ) $180 \mathrm{mg} / \mathrm{m}^{2}$ was given intravenously on the first day, then Calcium folinate (CF) $200 \mathrm{mg} / \mathrm{m}^{2}$; CF $200 \mathrm{mg} / \mathrm{m}^{2}$ was given intravenously on the first and second day; 5 -FU $400 \mathrm{mg} / \mathrm{m}^{2}$ was given in bolus immediately after CF on the first and second day; 5 -FU $600 \mathrm{mg} / \mathrm{m}^{2}$ was given continuously after bolus for 22 hours on the first and second day. Bevacizumab 5 $\mathrm{mg} / \mathrm{kg}$ was given intravenously before chemotherapy. 5HT3 receptors blocking agent were used before chemotherapy to prevent nausea and omit. Atropine $0.1 \mathrm{mg}$ was given subcutaneously once the cholinergic syndrome appeared. Loperamide hydrochloride was given immediately two tablets per time once the delayed diarrhea occurred, and then given one tablet per two hours, till 12 hours after the last looserstools, the total amount of loperamide was no more than 12 tablets. A cycle was two weeks, the evaluation in short result was done after 4 cycles, remission or stable disease need continue treatment until disease progression or intolerance. Before treatment, all patients signed the informed consent, tube
PICC was inserted in parallel.

\subsection{Evaluation Criteria}

The recent results were determined by the short-term effect evaluation criteria of solid tumors by World Health Organization (WHO), the evaluation criteria are as follow: complete remission, CR, partial remission, PR, stable disease, SD, progressive disease, PD. Clinical benefit rate was calculated through the result of $\mathrm{CR}+\mathrm{PR}+\mathrm{SD}$, Efficacy evaluation was based on physical examination, $\mathrm{X}$ ray, CT and other tests, followed regularly and confirmed after 4 weeks. The median time to progression (mTTP) was as the long-term indicator to evaluate the efficacy. The progression time was from the start of treatment until after the time of tumor progression. Adverse reactions were evaluated by NCI-CTC (version 2) standard evaluation, which divided into 0-IVdegrees.

\section{Results}

\subsection{Efficacy Evaluation}

All the patients were treated with a total of 93 treatment cycles, with an average of 6.2 treatment cycles for per patient. All the patients can be carried out evaluation of the efficacy and toxicity. Short-term efficacy was evaluated after 4 cycles of therapy, results were as flows: CR 0\%, PR 66.7\%, SD 20\%, PD 13.3\%, clinical benefit rate 86.7, mTTP 10.6 months.

\subsection{Toxicity}

Main toxicity were marrow suppression, cholinergic syndrome and delayed diarrhea, they were all listed in Table 1, No treatment-related death occurred to all the patients.

\section{Discussion}

We have adopted the combination of FOLFIRI program and anti-angiogenesis drugs Bevacizumab, discussed the efficacy and safety of the chemotherapy plus Becacizumab as therapy for Chinese patients with advanced colorectal cancer.

A big step forward in the treatment of advanced colorectal cancer is the emergence and application of monoclonal antibody drugs, which mainly included Bevacizumab for vascular endothelial growth factor (VEGF) and Cetuximab for epidermal growth factor receptor (EGFR). A number of studies have showed that, the over-expression of VEGF is closely related to the tumor invasiveness, vascular density, metastasis and recurrence and prognosis. Bevacizumab is a new anti-VEGF recombinant humanized monoclonal antibody. It plays a role in anti-angiogenesis primarily through neutralizating the VEGF and blocking its receptor-binding of the surface of endothelial 
Table 1. The toxicity of Bevacizumab plus FOLFIRI regimen as front-line therapy (case).

\begin{tabular}{|c|c|c|c|c|c|}
\hline Side effects & 0 degree & I degree & II degree & III degree & V degree \\
\hline Hemoglobin & 12 & 2 & 1 & 0 & 0 \\
\hline Leukocyte & 10 & 3 & 2 & 0 & 0 \\
\hline Platelet & 10 & 4 & 1 & 0 & 0 \\
\hline Liver function & 9 & 4 & 1 & 1 & 0 \\
\hline Cretonne & 13 & 1 & 1 & 0 & 0 \\
\hline $\begin{array}{l}\text { Nausea and } \\
\text { vomiting }\end{array}$ & 8 & 5 & 1 & 1 & 0 \\
\hline $\begin{array}{l}\text { Cholinergic } \\
\text { syndrome }\end{array}$ & 13 & 1 & 1 & 0 & 0 \\
\hline Delayed diarrhea & 10 & 1 & 2 & 2 & 0 \\
\hline Constipation & 12 & 2 & 1 & 0 & 0 \\
\hline Rash & 13 & 1 & 1 & 0 & 0 \\
\hline Hypertension & 10 & 4 & 1 & 0 & 0 \\
\hline Bradycardia & 14 & 1 & 0 & 0 & 0 \\
\hline $\begin{array}{l}\text { Non-infectious } \\
\text { fever }\end{array}$ & 13 & 2 & 0 & 0 & 0 \\
\hline
\end{tabular}

cells. In addition, it can also reduce tumor interstitial fluid pressure in favor of chemotherapy drugs reaching the tumor site to play a role in anti-tumor [4]. Avastin has been approved in combination with chemotherapy for non-small cell lung cancer, colorectal cancer, breast cancer and other patients with advanced by FDA [4]. Eastern Cooperative Oncology Group conducted a randomized controlled phase III study receiving 829 patients with advance colorectal cancer who have experienced the failure treatment of combination of Irinotecan and Fluorouracil previously; they were randomly divided into 3 groups: the combination therapy of FOLFOX4 and Bevacizumab group, FOLFOX4 group and Bevacizumab monotherapy group. Bevacizumab $10 \mathrm{mg} / \mathrm{kg}$ was given by intravenous drip one time per 2 weeks, results showed that the median survival time of chemotherapy plus Bevacizumab group was 12.9 months, it was significantly longer than the chemotherapy group the median survival time of which was 10.8 months $(\mathrm{HR}=0.75 ; \mathrm{P}=0.0011)$, The objective response of recent clinical evaluation also had significant increase $(22.7 \%$ vs. $8.6 \%, \mathrm{P}<0.0001)$ [5]. Bevacizumab can not only improve the second-line treatment effects in combination with chemotherapy, it also further improves the survival of patients in combination with the first-line chemotherapy. Some researchers have evaluated the efficacy and safety of FOLFOX4 regimen combined with Bevacizumab as first-line treatment of metastasis colorectal cancer. Bevacizumab $5 \mathrm{mg} / \mathrm{kg}$ was given by intravenous drip one time per 2 weeks, among 53 patients: 8 cases CR (15.1\%), 28 cases PR 28 (52.8\%), objective response rate $67.9 \%, 11$ cases SD (20.7\%), 6 patients (11.3\%) disease progression. Median follow-up 13.5 months, TTP 11 months, predicated 1, 2 and 3-year survival rates were $79.8 \%, 63.8 \%$ and $58.3 \%$ respectively. 2 patients relapsed during follow-up. 8 patients (15\%) achieved RO resection of metastasis [6]. Saltz etc. reported 1401 patients with advanced colorectal cancer were randomly divided into 2 groups, Capecitabine and Oxaliplatin in XELOX group and FOLFOX4 group, then each further divided into 2 groups, plus Bevacizumab group and placebo group, the primary endpoint was PFS. The results showed that the PFS of Bevacizumab group was 9.4 months significantly longer than the placebo group with 8 months PFS $(P=0.0023)$ [7]. Besides in combination with the Oxaliplatin-based chemotherapy, researchers also explored the joint application of Bevacizumab and Irinotecan-based chemotherapy. In a Phase III clinical study, 923 previously untreated patients with advanced colorectal cancer were randomly divided into IFL/placebo group, IFL/Bevacizumab group and FU/ LV/Bevacizumab group, Bevacizumab $5 \mathrm{mg} / \mathrm{kg}$ was given intravenously, one time per two weeks. Once the safety of IFL/Bevacizumab group was determined by the interim analysis, FU/LV/Bevacizumab group stopped into the group. Results display that the median survival time of Bevacizumab combined IFL group was significantly longer than the IFL group, and the median survival time trend of Bevacizumab combined FU/LV group was longer IFL group, suggesting that on the basis of IFL adding Bevacizumab can significantly prolong the survival time of initial treatment patients with advanced colorectal. For those patients not suitable for the application of Irinotecan, given Bevacizumab on the basis of $\mathrm{FU} / \mathrm{LV}$ is also an active choice [8]. However, in this study, Fluorouracil in the combined chemotherapy was given through bolus administration, as mentioned above; this method is not the best way of co-administration. Therefore, we choose the FOLFIRI regimen consist of Irinotecan and continuous infusion of Fluorouracil as first-line chemotherapy program, on this basis, plus this anti-tumor angiogenesis drug Bevacizumab, the results showed better efficacy, recent efficiency reached 66.7\%; clinical benefit rate was up to $86.7 \%$, median time to progression was 10.6 months, they were close to the foreign literature [9]. It should be noted that the dosage of Bevacizumab in part of the foreign study was larger, in this study group was $5 \mathrm{mg} / \mathrm{kg}$, and it can also achieved similar clinical efficacy. For the dosage of such antitumor angiogenesis drug, it is worth further research $[5,10]$.

As reported in the literature, the main side effects of Irinotecan were abdominal pain, diarrhea, and bone mar- 
row suppression. The first two ones can be either part of the cholinergic syndrome or occurred in the form of delayed diarrhea after 24 hours. Acute cholinergic syndrome not only include the early diarrhea and abdominal pain, but also the sweating, contracted pupil, tearing, blurred vision, dizziness and low blood pressure, the incidence of such performances was $9 \%$ as reported [11]. The incidence is this group was similar to related report, indicating that the addition of Bevacizumab did not increase the incidence of this adverse reaction. 5 patients in this group suffered from delayed diarrhea, and only 2 patients with III degree adverse reaction, which were much lower than reported in literature abroad [11]. As it will be related with patients and medicines from different sources, the small sample and timely application of Loperamide may also be a factor. The main side effects of Bevacizumab were hypertension, gastrointestinal perforation, and increased risk of bleeding and therefore not recommended for elderly patients with hypertension and the patients proposed to receive recent surgical treatment. During the treatment in this group, blood pressure changes had been closely monitored, hypertension appeared in 2 cases with normal blood pressure, the blood pressure increased further in 3 cases of primary hypertension patients, all presented as diastolic blood pressure, and they all had been controlled through the adjustment of antihypertensive drugs, no gastrointestinal perforation and bleeding occurred in the patients during the treatment.

The present study showed that after 4 cycles of therapy with Bevacizumab plus FOLFIRI regimen treating Chinese patients with advanced colorectal cancer, 1) the short-term efficacy was evaluated as flows: CR $0 \%$, PR $66.7 \%$, SD $20 \%$, PD $13.3 \%$, clinical benefit rate 86.7 , and mTTP 10.6 months; 2) there was a little drug toxicity such as marrow suppression, cholinergic syndrome and delayed diarrhea, and no treatment-related death occurred. The data suggested that the therapy with Bevacizumab plus FOLFIRI regimen treating Chinese patients with advanced colorectal cancer was better than only one anticancer drug treatment or the combination treatment with Irinotecan plus Fluorouracil.

In summary, this study showed that anti-tumor angiogenesis drugs Bevacizumab combined with Irinotecanbased FORFIRI regimen as the first-line treatment of advanced colorectal cancer, they can obtain good effect for patients in China, and the side effects of this treatment can be controlled and handled, be worthy of further application.

\section{Acknowledgements}

This work was supported by Xinxiang Medical Univer- sity and Kamp Institute for Medical Research.

\section{REFERENCES}

[1] H. Y. Luo, Y. H. Li, L. Zhang, W. Q. Jiang, Y. X. Shi, F. Wang, Y. J. He and R. H. Xu, "Efficacy of CPT-11 Combined 5-FU/CF (FOLFIRI) Regimen on Advanced Colorectal Cancer,” Chinese Journal of Cancer, Vol. 26, No. 8, 2007, pp. 905-908.

[2] J. Li, "Application of Monoclonal Antibody in Combination with Irinotecan in the Treatment of Colorectal Cancer,” Chinese Journal of Oncology, Vol. 28, No. 10, 2006, pp. 796-797.

[3] J. Ma, S. K. Qin and Q. Y. Zhang, "Album of Chinese Education in Clinical Oncology,” Heilongjiang Science and Technology Press, Haerbin, 2007, pp. 302-306.

[4] S. P. Khosravi and P. I. Fernández, "Tumoral Angiogenesis: Review of the Literature,” Cancer Investigation, Vol. 26, No. 1, 2008, pp. 104-108. doi:10.1080/07357900701662509

[5] B. J. Giantonio, P. J. Catalano, N. J. Meropol, P. J. O’Dwyer, E. P. Mitchell, S. R. Alberts, M. A. Schwartz and A. B. Benson, "Bevacizumab in Combination with Oxaliplatin, Fluorouracil, and Leucovorin (FOLFOX4) for Previously Treated Metastatic Colorectal Cancer: Results from the Eastern Cooperative Oncology Group Study E3200,” Journal of Clinical Oncology, Vol. 25, No. 12, 2007, pp. 15391544.

[6] C. Emmanouilides, G. Sfakiotaki, N. Androulakis, K. Kalbakis, C. Christophylakis, A. Kalykaki, L. Vamvakas, A. Kotsakis, S. Agelaki, E. Diamandidou, N. Touroutoglou, A. Chatzidakis, V. Georgoulias, D. Mavroudis and J. Souglakos, "Front-Line Bevacizumab in Combination with Oxaliplatin, Leucovorin And 5-Fluorouracil (FOLFOX) in Patients with Metastatic Colorectal Cancer: A Multicenter Phase II Study,” BMC Cancer, Vol. 7, 2007, p. 91.

[7] L. B. Saltz, S. Clarke, E. Díaz-Rubio, W. Scheithauer, A. Figer, R. Wong, S. Koski, M. Lichinitser, T. S. Yang, F. Rivera, F. Couture, F. Sirzén and J. Cassidy, "Bevacizumab in Combination With Oxaliplatin-Based Chemotherapy as First-Line Therapy in Metastatic Colorectal Cancer: A Randomized Phase III Study,” Journal of Clinical Oncology, Vol. 26, No. 12, 2008, pp. 2013-2019.

[8] H. I. Hurwitz, L. Fehrenbacher, J. D. Hainsworth, W. Heim, J. Berlin, E. Holmgren, J. Hambleton, W. F. Novotny and F. Kabbinavar, "Bevacizumab in Combination with Fluorouracil and Leucovorin: An Active Regimen for First-Line Metastatic Colorectal Cancer,” Journal of Clinical Oncology, Vol. 23, No. 15, 2005, pp. 3502- 3508.

[9] H. Hurwitz, L. Fehrenbacher, W. Novotny, T. Cartwright, J. Hainsworth, W. Heim, J. Berlin, A. Baron, S. Griffing, E. Holmgren, N. Ferrara, G. Fyfe, B. Rogers, R. Ross and F. Kabbinavar, "Bevacizumab Plus Irinotecan, Fluorouracil, and Leucovorin for Metastatic Colorectal Cancer," The New England Journal of Medicine, Vol. 350, 2004, pp. 2335-2342.

[10] D. R. Gandara, R. Sangha and A. M. Davies, "Bevacizu- 
mab: Optimal Dose, Schedule, and Duration of Therapy,” Clinical Lung Cancer, Vol. 8, No. 9, 2007, pp. 522-523.

[11] C. Tournigand, T. André, E. Achille, G. Lledo, M. Flesh, D. Mery-Mignard, E. Quinaux, C. Couteau, M. Buyse, G. Ganem, B. Landi, P. Colin, C. Louvet and A. de Gramont,
"FOLFIRI Followed by FOLFOX6 or the Reverse Sequence in Advanced Colorectal Cancer: A Randomized GERCOR Study,” Journal of Clinical Oncology, Vol. 22, 2004, pp. 229-237. 